\title{
Improving the Usability and Effectiveness of Online Learning: How Can Avatars help?
}

\author{
Hua Wang1,2, Mark Chignell ${ }^{1}$, Mitsuru Ishizuka ${ }^{2}$ \\ ${ }^{1}$ University of Toronto, \\ \{hwang, chignel\}@mie.utoronto.ca \\ ${ }^{2}$ University of Tokyo, 7-3-1 Hongo, Bunkyo-ku \\ Tokyo 133-0033, Japan \\ \{ishizuka\}@miv.t.u-tokyo.ac.jp
}

\begin{abstract}
This paper describes Empathic Tutoring System (ETS) which uses character agents for online learning. Eye movement tracking and other physiological measures are used to personalize character agent behaviors (affective and instruction) in an e-learning environment. A prototype system reacts to learner's eye information in real-time, recording eye gaze and pupil dilation data (plus heart rate and skin conductance) during learning. Based on these measures, character agents inferred the attentional and motivational status of the learner and responded accordingly with affective and instructional behaviors. Character agents engage and direct the learner's attention while providing both generalized system help and personalized advice about the learning content. Observations from usability studies with ETS suggest that e-learning character agents reacting to eye gaze and physiological measures may heighten learner concentration and lead to more effective learning.
\end{abstract}

\section{INTRODUCTION AND MOTIVATION}

In an increasingly complex world, learning of all kinds is at a premium. Increasingly, life-long learning is becoming a goal, as people have to adjust to changes in jobs and technologies. Education is costly and e-learning has shown the potential to provide a broad range of training at a possibly lower cost. Among the possible advantages of an e-learning approach are the ability to present educational content any time and anywhere. However, the traditional classroom has many advantages, including the presence of a skillful teacher who can motivate students, diagnose problems, and personalize instruction.

In traditional educational settings, good teachers recognize learning needs and learning styles and adjust the selection and presentation of content accordingly. In online learning, there is a need to create more effective interaction between online educational content and learners that recaptures some of the benefits of interacting with a good teacher, including the creation of a more natural and friendly environment for learning.

In this paper we review recent work on the development of software agents that can provide some of the functions normally performed by a human teach and propose a more systematic approach to the development of e-learning avatars based on ergonomic principles. Avatars are visualizations of agents. Avatars may be distinguished between those that represent real people, and those that represent software agents. For instance, people interacting in a virtual environment would be represented by the first form of avatar. Conversely, a news-reading avatar like "Ananova" is not meant to represent a real person but instead to represent a software agent or functionality. In this paper we will focus on the second form of avatar and how it may be used effectively in e-learning. As of this writing, there are a number of tools that assist in implementing avatars. In the systems that we have constructed, characters are controlled by a version of MPML [1]. Using a tool such as this, the avatar may interact with learners using synthetic speech and gestures.

We will focus on the following two functions for e-learning avatars: a) increasing motivation by stimulating interest in online learning; b) Detecting learning styles and modifying instructional strategies to fit different learning patterns.

\section{RELATED WORK}

Recent research has studied the use of life-like character as surrogate teachers. Lester et al. in their COSMO system used a life-like character that teaches how to treat plants [2]. Conati used a probabilistic learner model [3], based on a dynamic decision network, to represent the affective state of the learner interacting with an educational game, as well as to represent the learner's personality and goals. Johnson [4] used character agents and eye-tracking for foreign language/culture training. $\mathrm{Qu}$, et al. [5] described a system that took account of the attention of the learner, and the expected time required to perform task, to choose when to interact with learners. 


\section{METHODODY}

\section{WHAT EDUCATIONAL AVATARS KNOW AND DO}

\section{Presenting Content}

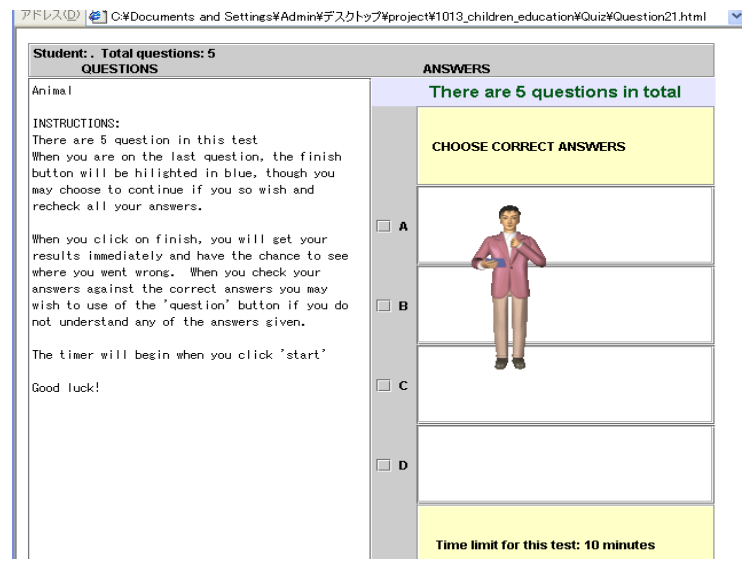

Figure 1. Quiz Interface.

One of the main tasks of the avatar is to administer quizzes and provide personalized responses. The avatar may act as a tutor and display empathic emotions (Figure 1). Multimedia elements such as figures, flash animations, and video clips may be used to illustrate the instructional material (Figure 2).

\section{Showing Emotions}

Cooper et al. [6] argued that showing emotions, empathy and understanding through facial expressions and body language is essential in tutor-learner and learner-learner interaction. Children who received sympathy from the experimenter after failing at a task, tended to attribute that failure to their lack of ability, while children who received mild anger tended to attribute their failure to lack of effort [7].

This finding can be incorporated into an avatar that performs several functions/behaviors including the displaying different types of emotional reaction. For instance, an avatar may show a happy/satisfied emotion if the learner concentrates. In contrast, an avatar may show mild anger or alert learner who seem to lose concentration. The agent may also show empathy when the learner is stuck or gives a wrong answer.

\section{Awareness of learners}

Real teachers are able to read body language and understand the psychology of the learner to some extent. e-Learning avatars may also benefit from an awareness of user states and the ability to customize and adapt instruction accordingly. For instance, an avatar may be "eye-aware", using eye movements, pupil dilation, and changes in overall eye position (head movements) to make inferences about the state of the learner that guide its behavior. After determining the learner's eye position information and current area of interest or concentration, the agents can move around to highlight the current learning topic, in order to attract or focus the learner's attention. For instance, with eye gaze data, agents react to Eye information in real time through actions such as moving to the place being looked at or by showing the detailed information content for where learners are looking at, etc.
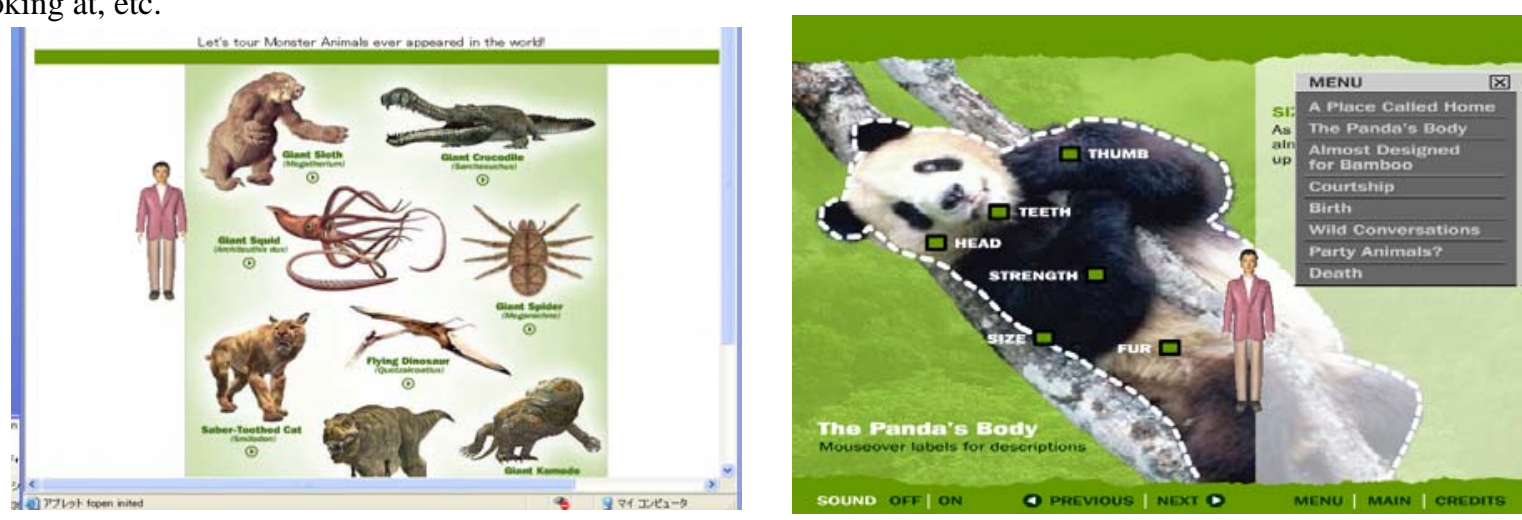
Figure 2. Presentation of Biology Content.

Eye movement analysis can be used to determine where learners look and how long they look at different objects. Applications using eye-tracking can be diagnostic or interactive [8]. In diagnostic use, eye movement data provides evidence of the learner's focus of attention over time and can be used to evaluate the usability of interfaces. In interactive use, a system responds to the observed eye movements which can thus serve as an input modality.

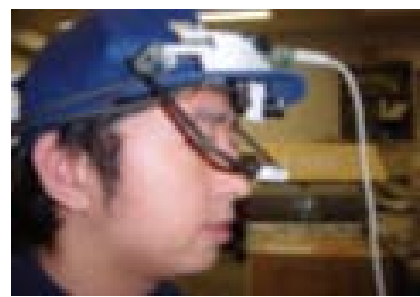

Figure 3. Eye Tracker.

Figure 3. shows an example of a eye tracker where the cameras that read eye information are mounted on a cap. With CCD cameras becoming increasingly inexpensive, it may be possible to place several such cameras around a monitor so that eye tracking is completely unobtrusive other than in its effects.

\section{Providing Motivation}

The character Agent can provide motivation as well as feedback or instruction. The feedback can be based on various measures of user state, including general activity (e.g., keys pressed and mouse actions, and physiological indicators of arousal and stress.) As an example of motivational output, an avatar may remind learners to concentrate on a topic if they repeatedly look away from the screen/content. When learners show interest in the content the character agents may provide encouraging responses. However, if a learner shows less interest (as indicated by physiological measures such as reduced levels of pupil dilation) then the agent might inquire if the learner is tired or bored. Boredom or fatigue may also be inferred based on lower overall activity in terms of mouse clicks and key selections. Providing rest time or changing topics are other strategies that character agents can use to deal with situations where the learner appears to be bored or fatigued.

Figure 4 shows how a character agent may react to feedback about the learner's status based on eye-tracking information. In this example, the eye tracker collects eye gaze information and the system then infers what the learner is currently attending to (focusing on). This information is then combined with the learner's activity records and an appropriate pre-set strategy is selected. The character agent then provides feedback to the learner, tailoring the instructions and emotions (e.g., facial expressions) to the situation.

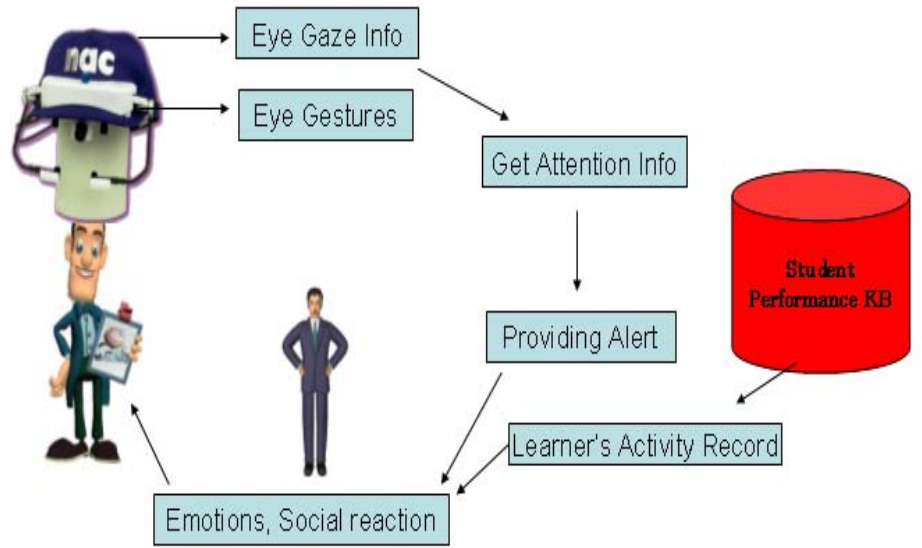

Figure 4. Real-Time Use of Eye Information in ETS. 


\section{EXAMPLES OF USE}

\section{IMPLEMENTATION APPROACHES}

We used the Microsoft Agent Engine to create the characters and also the emotions, movements and control functions, using the MPML agent control language. Other approaches might also have been used. For instance, Huang, et al. [9] developed a character agent that interacted with people while they were browsing Web pages. Their system was constructed mainly from Java, JavaScript and XML. In ETS, eye gaze data is stored and transferred using an XML file. The serial Data is transferred to an XML file using the Com port and the information is then sent to the interface using JavaScript. During this process, the eye data is mapped to the screen position.

The Eye Marker eye tracking system was used to detect eye information (with the basic data being collected using 2 cameras facing towards the eye. In a preliminary study participants were found to look more carefully at the learning content (and listen more carefully to the agent) when they were provided with real time feedback about their eye positions.

We have investigated the areas that participants look at when interacting with an avatar, along with their eye traces. (Figure 5). The figure shows an example of the areas that people paid attention to. We calculated the number of eye gazes in each area and how the eye trace moved. Of particular interest were eye movement patterns where the learner's eye traces went to the character agent and then back to the learning content. This type of interaction showed that the learners could receive feedback from the character agent and could then return to the current topic without losing track of where they were. Analysis of the data showed that the eye position moves with the focus of the current topic fairly well showing that the learners were concentrating on the content being taught.



Figure 5 Eye Traces on different areas

\section{Single-Avatar VS Multiple-Avatar}

As shown in Figure 6, learners using both interfaces give more attention to the knowledge content. But at the same time, in the multiple avatar interface, the eye positions are more focused on current topics and this may indicate that the multiple avatars may help the learner to know their situation better and thus improve the learning process. 


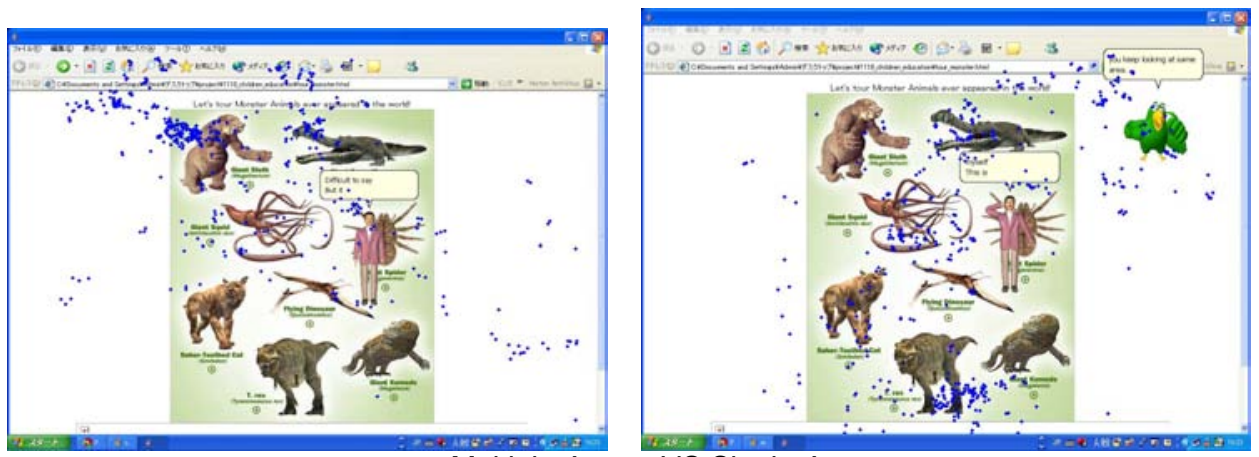

Figure 6Multiple-Avatar VS Single-Avatar

Experiment

We are currently running an experiment that examines how learners respond to different avatar behaviours. In particular we are focusing on if and how eye aware avatars vs. non-eye aware avatars facilitate learning. The results of this experiment will be reported in the final version of this paper should it be accepted for publication in the HFES annual meeting.

\section{DISCUSSION AND FUTURE WORK}

By getting information about learner response (such as eye movement data), avatar interfaces can interact with learners more effectively and provide appropriate feedback. From a preliminary assessment of usability, avatars seem to have a beneficial effect on learner motivation and concentration during learning. We are currently running formal experiments to that will test this hypothesis more formally. Further experimental research will be required to determine when and how agent-based interaction can supplement the beneficial social interactions that occur with human teachers, tutors, and fellow students within a learning community. Of particular interest will be if an how eye trackers can enhance the funcationality and usefulness of e-Learning avatars.

In early evaluations of avatar mediated e-Learning, we have found that learners tended to pay attention to the explanation from character agents and also found feedback about their own eye movements useful. They felt that they looked at the contents more attentively with eye tracking feedback and that this feedback made them more engaged with the learning content. As eye tracking technology improves it will become more convenient to use and will provide increasingly precise feedback, which may lead to the development of more sophisticated and socially aware avatars. The use of multiple character agents within empathic tutoring systems should also be an interesting future direction for this type of research.

\section{ACKNOWLEDGMENTS}

We would like to thank Dr. Alan Kay for his advice and suggestions with respect to this project.

\section{REFERENCES}

1. Prendinger, H., Descamps. S., and Ishizuka, M., MPML: A markup language for controlling the behavior of life-like characters. Journal of Visual Languages.

2. Stone, B., and Lester, J., Dynamically Sequencing an Animated Pedagogical Agent, Proceedings of the Thirteenth National Conference on Artificial Intelligence, Portland, OR, pp. 424-431, 1996.

3. Conati, C., Chabbal, R. and Maclaren, H. A Study on using biometric sensors for monitoring learner emotions in educational games. Workshop on Assessing and Adapting to Learner Attitudes and Affect: Why, When and How?, in conjunction with Learner Modeling (UM-03), 2003.

4. Johnson, W. L., Choi, S., Marsella, S., Mote, N., Narayanan, S. and Vilhjálmsson, H., Tactical Language Training System: Supporting the Rapid Acquisition of Foreign Language and Cultural Skills Proceedings of InSTIL/ICALL NLP and Speech Technologies in Advanced Language Learning Systems, 2004

5. Qu, L., Wang, N. and John, W.L., "Choosing when to interact with learners", Proceedings of the 9th international conference on Intelligent user interface table of contents,Funchal, Madeira, Portugal, Short Papers table of contents, pp 307-309,2004. 
6. Cooper B, Brna P, Martins A. Effective Affective in Intelligent Systems - Building on Evidence of Empathy in Teaching and Learning. In: Affective Interactions: Towards a New Generation of Computer Interfaces. Paiva A ed. London: Springer Verlag. 2000

7. Palloff, R. M. and Pratt, K., Lessons from the cyberspace classroom: The realities of online teaching. San Francisco: Jossey-Bass., 2001

8. Duchowski, T.,.Eye Tracking Methodology: Theory and Practice. Springer, London, UK, 2003.

9. Huang, Z., Elis, A. and Visser, C., Facial Expressions for

Embodied Agents in STEP, Proceedings of AAMAS 2004 Workshop on Embodied Conversational Agents: Balanced Perception and Action, 2004. 